\title{
Salivary IgA enhancement strategy for development of a nasal-spray anti-caries mucosal vaccine
}

\author{
YAN HuiMin \\ Mucosal Immunity Research Group, State Key Laboratory of Virology, Wuhan Institute of Virology, Chinese Academy of Sciences, \\ Wuhan 430071, China
}

Received March 10, 2013; accepted March 21, 2013

\begin{abstract}
Dental caries remains one of the most common global chronic diseases caused by Streptococcus mutans, which is prevalent all over the world. The caries prevalence of children aged between 5-6 years old in China is still in very high rate. A potent and effective anti-caries vaccine has long been expected for caries prevention but no vaccines have been brought to market till now mainly due to the low ability to induce and maintain protective antibody in oral fluids. This review will give a brief historical retrospect on study of dental caries and pathogenesis, effective targets for anti-caries vaccines, oral immune system and immunization against dental caries. Then, salivary IgA antibodies and the protective responses are discussed in the context of the ontogeny of mucosal immunity to indigenous oral streptococcal. The methods and advancement for induction of specific anticaries salivary sIgA antibodies and enhancement of specific anti-caries salivary sIgA antibodies by intranasal immunization with a safe effective mucosal adjuvant are described. The progress in the enhancement of salivary SIgA antibodies and anticaries protection by intranasal immunization with flagellin-PAc fusion protein will be highlighted. Finally, some of the main strategies that have been used for successful mucosal vaccination of caries vaccine are reviewed, followed by discussion of the mucosal adjuvant choice for achieving protective immunity at oral mucosal membranes for development of a nasal-spray or nasal-drop anti-caries vaccine for human.
\end{abstract}

mutans streptococci, secretory IgA, PAc, mucosal immunization, caries vaccine

Citation: Yan H M. Salivary IgA enhancement strategy for development of a nasal-spray anti-caries mucosal vaccine. Sci China Life Sci, 2013, 56: 406-413, doi: 10.1007/s11427-013-4473-5

Dental caries is a chronic, infectious disease that results in localized dissolution and destruction of the calcified tissue mainly by Streptococcus mutans (S. mutans) [1-3]. This disease is one of the most common global chronic diseases caused by bacterial infection, which is prevalent in developed, developing, and underdeveloped countries and is distributed unevenly among the populations. The disease usually develops slowly and can occur throughout life, which affects children, adolescents, as well as adults. Furthermore, it is still a major oral health problem in most industrialized countries, affecting $60 \%-90 \%$ of schoolchildren and the

email: hmyan@wh.iov.cn vast majority of adults. It is also a most prevalent oral disease in China [4,5]. According to the third national epidemiological investigation on oral diseases in China conducted in 2005 , the caries prevalence rate of children aged between 5-6 years old remains as high as $66 \%$, people of 35-44 and 65-74 years old reach $61 \%$ and $75.2 \%$ respectively [6]. More than $98 \%$ of Chinese elder population has root-surface caries.

Anti-caries vaccine has long been expected for caries prevention since the early fifties of the 20th century. Many kinds of vaccine immunogens such as protein, recombinant or synthetic peptide, protein-carbohydrate conjugate, or DNA-based active vaccines and mucosal adjuvants such as 
heat-labile enterotoxins (HLT) from Vibrio cholera (LT-I) or Escherichia coli (LT-II), bupivacaine, chitosan have been successful in animal models [7-12]. However, no vaccines have been brought to market till now mainly due to the low ability to induce and maintain protective antibody in oral fluids. New mucosal immunization strategies and safe mucosal adjuvants have drawn attention for safe and effective anti-caries vaccine, which is facilitating the development of anti-caries vaccine from research bench to clinical use.

Flagellin as the ligand of toll-like receptor 5 (TLR5) has been proven to be an effective mucosal adjuvant and become promising for clinical use [13-19]. We applied the recombinant flagellin as mucosal adjuvant in anti-caries vaccines by nasal immunization, either using flagellin directly by simple mixing with antigen or using fusion strategy to combine flagellin and the target antigen in a single fusion protein, proved the efficacy for enhancement of specific $\operatorname{IgA}$ response in oral fluids and better protection against caries [11,20,21]. Here, I review some of the main strategies that have been used for successful mucosal vaccination of caries vaccine and discuss the importance of the mucosal adjuvant choice for achieving protective immunity at oral mucosal membranes.

\section{Dental caries and pathogenesis}

The pathogenesis of caries had been studied for decades. S. mutans, Streptococcus sobrinus (S. sobrinus) and their relatives, collectively known as the mutans streptococci, have been affirmed to be the major causative agents of dental caries in humans [2,3]. Serotype c $S$. mutans strains are most frequently isolated from the human oral cavity [3]. These bacteria are usually endogenous in adults and can be transferred from mother to child and are present at varying levels in all human mouths [22,23]. Both $S$. mutans and $S$. sobrinus produce water-soluble and water-insoluble glucans from sucrose, by the combined action of glucosyltransferases, which is necessary for the accumulation of bacteria cells on the tooth surface. The bacteria adhere and congregate on the surface of tooth, then the metabolized lactate lead to decalcification in enamel, followed by damage in dentin and dental pulp [24].

Currently used strategies for caries prevention, such as oral health education, chemical and mechanical control of plaque, use of fluorides, and application of pit and fissure sealants, are mostly broadly effective [25]. However, economic, behavioral, or cultural barriers to their use have continued the epidemic of dental disease in the mouths of many people on a global level. Furthermore, currently used strategies for treatment of the disease are largely limited to removal of the diseased part of the tooth and placing a suitable restoration, which is of high cost but not able to eradicate caries on other teeth. Although this disease has attracted increasing attention in recent decades, scant attention is paid to controlling the disease itself. Immunization programs have led to the elimination and/or control of different infectious diseases, including smallpox, polio, measles, mumps, rubella, Haemophilus influenza type B disease, pertussis, tetanus, and diphtheria. Development of an effective anti-caries vaccine is becoming urgently required as the final best way to prevent caries in the future.

\section{Effective targets for anti-caries vaccines}

The very initial studies on dental caries vaccines applied whole cells of mutans streptococci as a possible vaccine $[26,27]$. Thereafter, various cell-surface antigens of mutans streptococci and their recombinant fragments or even synthetic peptides have been studied as possible candidates for dental caries vaccines [28,29]. In the recent decade, attention has become focused on three protein antigens: the surface fibrillar adhesins, the glucosyltransferases (GTF) and the glucan-binding proteins, all of which have demonstrable associations with virulence and the process of tooth surface colonization $[8,12,30-32]$. It is demonstrated that the streptococcal surface fibrillar adhesins control attachment to tooth surfaces, the glucosyltransferases produce adhesive glucans from sucrose, and the cell-wall-associated glucan-binding proteins have the ability to bind $\alpha-1,6$-glucan and may provide the receptors for glucan-mediated aggregation [33]. These components can be utilized as effective targets for anti-caries vaccines.

\subsection{Adhesins}

The streptococcal surface adhesin proteins as effective antigenic components have been obtained from $S$. mutans (variously identified as antigen I/II, PAc, or P1) and S. sobrinus (SpaA or PAg). Abundant in vitro and in vivo evidence indicates that antibody with specificity for $S$. mutans PAc or S. sobrinus SpaA can interfere with bacterial adherence and subsequent dental caries [33]. PAc has been utilized in experimental systems and proven to be a most effective immunogen for caries vaccine development in many forms such as in full-length protein, recombinant or synthetic peptide [34], protein-carbohydrate conjugate [35], or DNA-based active vaccines [12].

\subsection{Glucosyltransferases (GTFs)}

S. mutans and S. sobrinus each synthesize several glucosyltransferases. The activity of GTF is mediated through both catalytic and glucan-binding functions. The presence of antibody to glucosyltransferase in the oral cavity prior to infection can significantly influence the disease outcome, presumably by interference with one or more of the functional activities of the enzyme. The antibody directed to native GTF or sequences associated with its catalytic or 
glucan-binding function interferes with the synthetic activity of the enzyme and with in vitro plaque formation $[33,36]$. Thus GTF has also been utilized in experimental systems and proven to be an effective immunogen for caries vaccines.

\subsection{Glucan-binding proteins (GBP)}

At least three $S$. mutans glucan-binding proteins GbpA, $\mathrm{GbpB}$ and $\mathrm{GbpC}$ were identified to date [37]. Only GbpB, which is 431 residues long and has a calculated molecular weight of $41.3 \mathrm{kD}$, has been shown to induce protective immune responses to experimental dental caries.

\section{Oral immune system and immunization against dental caries}

Components of the oral immune system include the salivary glands, along with mucosal secretions, which contain an antibody called secretory immunoglobulin A, or "sIgA", which can survive in harsh environments such as digestive and respiratory tracts. Secretory $\operatorname{IgA}$ protects the entire body against a multitude of invading microbes, and is resistant to degradation caused by exposure to various enzymes. Due to the salivary secretions, this secretory immunoglobulin also coats and protects every tooth and helps protect them from harmful bacteria that may cause decay. Although SIgA antibody in saliva and other secretions is essentially absent at birth, mature sIgA, i.e., dimeric $\operatorname{IgA}$ with a bound secretory component, is the principal salivary immunoglobulin secreted in individuals by one month of age [38,39]. Children between 12 and 24 months exhibit a broader range of generally higher salivary $\operatorname{IgA}$ concentrations (median concentrations of $50-60 \mu \mathrm{g} \mathrm{mL}^{-1}$ ) than seen in the 1 st year [40]. The concentrations of $\operatorname{IgG}, \operatorname{IgA}$, and IgM in unstimulated human whole saliva are 14, 194 and 2 $\mu \mathrm{g} \mathrm{mL}{ }^{-1}$, respectively, indicating that secretory $\operatorname{IgA}(\operatorname{sIgA})$ is quantitatively the most important immunoglobulin in saliva $[41,42]$. Therefore, $\operatorname{sg} \mathrm{A}$ is the principal immune component of major and minor gland salivary secretions and considered to be the primary effector of adaptive immunity in the salivary milieu.

A large body of studies over several decades has demonstrated the feasibility of inducing protective immunity against mutans streptococci and the subsequent development of dental caries in animal models. More and more mucosal applications of dental caries vaccines have been sought. It is demonstrated that the anti-caries activity has been attributed to mutans streptococci-specific sIgA antibodies which can inhibit sucrose-independent or sucrosedependent mechanisms of streptococcal accumulation on tooth surfaces according to the choice of vaccine antigen. Therefore, the goal of immunizing infants and young children against colonization by mutans streptococci and hence diminishing the development of caries count on applying mucosal vaccination that would induce salivary $\operatorname{IgA}$ antibodies without the complications of parenteral injection [43].

Information from various small-scale human trials in adult volunteers has shown the applicability to increase levels of salivary sIgA antibodies to mutans streptococci, and in some cases to interfere with mutans streptococcal colonization. For example, oral administration of capsules containing glucosyltransferases (GTF) from S. sobrinus combined with aluminum phosphate could result in an increase in sIgA antibody response in 14 human subjects, which showed interference with proportions of indigenous mutans streptococci/total streptococcal flora, or total cultivable flora in the oral cavity $[44,45]$. Intranasal immunization of humans with $S$. mutans antigens could also induce an IgA response in secretions [37]. However, no vaccines have been brought to market though we are now in the fifth decade of vaccine approaches intended to prevent or arrest dental caries. This is mainly because of a low ability to induce and maintain protective antibodies in oral fluids [11].

\section{Salivary IgA antibodies and the protective responses}

Many clinical studies showed natural exposure to mutans streptococci results in a mucosal immune response to these organisms. Antibody activity to mutans streptococcal antigens can be detected in both saliva and sera, which was reviewed by Drs. Michalek and Childers [46].

Children often begin to synthesize serum antibodies to mutans streptococcal antigens mostly in the late 2 nd year of life, when mutans streptococci begin to accumulate on primary tooth surfaces. Then serum IgG antibody levels increase during childhood and remain detectable throughout life [40]. Prominent among the components reacting with IgA antibody are PAc, glucosyltransferase, and glucan-binding protein B. Studies that examined the association between serum antibody to $S$. mutans and dental disease in young adults and older adults showed different correlations between IgG antibody and their cumulative dental caries experience or disease levels. The various conclusions of these associative studies of antibodies to $S$. mutans in relation to dental caries experience reflect the complexity of immune response to natural exposure to mutans streptococci in different individuals. For detail of this subject, see review by Drs. Smith and Mattos-Graner [40].

Attempts to draw conclusions on the relationship between the levels or specificity of naturally formed salivary IgA antibody and dental disease in adults have also been problematic. Different investigations reported different relationships between levels of $\operatorname{IgA}$ antibody to mutans streptococci and caries experience. For detail of this subject, see review by Drs. Smith and Taubman [38]. As the mutans 
streptococci are usually endogenous and are present at varying levels in all human mouths, and the dental disease can occur throughout life and may progress under diverse environmental challenges, it is difficult to evaluate the protective efficacy of the saliva SIgA antibodies induced by natural exposure to mutans streptococci. Therefore, it is still not clear whether the naturally occurred $\operatorname{sIg}$ A against mutans streptococci is associated with protection against dental caries in man.

Taken together, we might deduce that the naturally occurred sIgA against mutans streptococci may not be strong enough or timely for providing protective effect against adhesion, colonization of the mutans streptococci and the following dental caries. Given that dental caries usually develops slowly and can occur throughout life, it may be anticipated that immune protection would need to be established prior to initial colonization or accumulation and be similarly long-lasting throughout life.

\section{Induction of specific anti-caries salivary sIgA antibodies}

The concept of vaccination against dental caries has existed almost from the time that this disease was recognized to result from colonization of the teeth by acidogenic bacteria. Unlike most other infectious diseases, the caries specifically occurs on teeth surfaces bathed by external secretions in which the principal immunoglobulin isotype present is $\operatorname{sgA}$. Therefore, immunization procedures which result in the induction of salivary $\operatorname{sgA}$ antibodies would most likely be effective means for inducing anti-caries immunity. Enormous strides have been made in comprehending the workings of the mucosal immune system by which sIgA antibodies are generated in saliva and other secretions [47]. Furthermore, many active immunization studies demonstrated the important anti-caries role of $\operatorname{sIgA}[43,48]$ though the relationship between the levels or specificity of naturally formed salivary $\operatorname{IgA}$ antibody and dental disease has not been clearly established as described above. The mechanisms of action of salivary sIgA antibodies against mutans streptococci include interference with their sucrose-independent and sucrose-dependent attachment to, and accumulation on, tooth surfaces, as well as possible inhibition of their metabolic activities [48]. Nevertheless, bacterial colonization or accumulation was targeted by vaccines for inactivation or blockage, rather than metabolic pathways critical to the survival of the microorganism. Thus, it is better to develop vaccines which can induce protective level of specific anti-caries salivary $\operatorname{sgA}$ antibodies to prevent initial colonization efficiently rather than to remove cariogenic microorganisms from an established biofilm.

Mucosal applications of dental caries vaccines are generally preferred for the induction of $\operatorname{sIgA}$ antibody in the salivary compartment, since mucosal IgA immune responses are most efficiently induced by the administration of vaccines onto mucosal surfaces while injected vaccines are generally poor inducers of mucosal immunity [49]. Many investigators have shown that exposure of antigen to mucosally associated lymphoid tissue in the gut, nasal, bronchial, or rectal site can give rise to immune responses not only in the region of induction, but also in remote locations. Though the basic principle of immune protection from dental caries caused by mutans streptococci has also been established in preclinical studies, refinements to the effective application of this approach to humans remain. Consequently, several mucosal routes have been used to induce protective immune responses to dental caries vaccine antigens.

Early studies with mucosally applied caries vaccines used the oral or intragastric route for antigen delivery. However, the oral route was not so ideal because the oral route requires antigen passage through the gut prior to uptake in the gut-associated lymphoid tissue (GALT) and the acidic stomach environment diminishes the effective antigen stimulus [40]. Topical applications of antigen to stimulate tonsil and minor salivary glands were also tried for inducing mucosal immune responses in the oral cavity. Even remote mucosal site, for example, rectal route was investigated for the ability to induce salivary $\operatorname{IgA}$ responses to mutans streptococcal antigens [33].

\section{Enhancement of specific anti-caries salivary sIgA antibodies by intranasal immunization with a safe effective mucosal adjuvant}

Different routes can lead to differential antigen presentation by specific dendritic cell (DC) subsets and induce differential antibody responses. In recent decade, more and more studies demonstrated that nasal vaccination is an effective regimen for the stimulation of antigen-specific protective immunity in both the mucosal $\mathrm{IgA}$ and serum $\mathrm{IgG}$ responses [50]. This route of mucosal immunization can elicit both humoral and cell mediated antigen-specific immune responses, and requires a much smaller dose of antigen than oral vaccination for the induction of antigen-specific mucosal and systemic immune responses. However, intranasal administration of vaccine antigen alone usually fails to fully stimulate NALT and induce mucosal and systemic immune responses. Thus to develop an effective NALT-targeted vaccine-antigen delivery system and a safe and effective adjuvant for intranasal administration with the vaccine antigen becomes a priority for development of intranasal mucosal vaccine.

Intranasal immunization has already been used to induce experimental immunity to antigens associated with mutans streptococcal colonization and accumulation. Protection via this route could be demonstrated with $S$. mutans PAc, GtfB, GbpB or GbpB-derived peptides [28,33], and even fimbrial 
preparations from $S$. mutans [51], using antigen alone or in combination with mucosal adjuvants. Specific saliva sIgA responses against $S$. mutans could also be elicited by intranasal immunization of pCIA-P/bupivacaine DNA complex $[7,8]$. The Fan group also tried to identify compounds and conditions that would increase antibody levels which could also improve the protective effect for anti-caries DNA vaccines since DNA vaccines are relatively weak immunogens [11]. This group demonstrated chitosan could increase salivary $\operatorname{IgA}$ antibody responses as adjuvants to a DNA vaccine of pGJA-P/VAX [12]. We sought to further enhance salivary $\operatorname{IgA}$ and serum $\operatorname{IgG}$ antibody responses by including Salmonella-derived flagellin protein at the time of pGJA-P/VAX administration $[11,20]$. We found that recombinant flagellin protein as a mucosal adjuvant for anti-caries DNA vaccine pGJA-P/VAX via intranasal immunization can increase serum PAc-specific IgG and saliva PAc-specific $\operatorname{IgA}$ antibody responses as compared with pGJA-P/VAX alone and significantly decrease the colonization of $S$. mutans on rat teeth. More importantly, flagellin protein as a mucosal adjuvant promoted the ability of pGJA-P/VAX to reduce caries lesions, providing effective protection against dental caries. It is demonstrated that recombinant flagellin protein as a mucosal adjuvant for intranasal caries DNA vaccine enhanced saliva IgA response and conferred better protection against caries [20].

Investigators have long sought to enhance mucosal IgA antibody responses by using different delivery systems such as liposomes, PLGA microparticles, ISCOMs or bioactive components as mucosal adjuvant such as Cholera toxin (CT), heat-labile enterotoxins (HLT) from Vibrio cholera (LT-I) or Escherichia coli (LT- II) [33]. These bioactive components can induce vigorous mucosal (and systemic) immune responses when used as adjuvants for protein antigens. However, their inherent toxicity precludes the use of such holotoxins in human vaccines, though work continues to identify safe HLT subcomponents which retain significant immunomodulatory characteristics that can be used at the preferred intranasal site $[10,11]$.

The field of vaccine adjuvants has been rapidly evolving since the discovery of the Toll-like receptor (TLR) family of pattern recognition molecules. The TLRs are membrane-bound receptors responsible for recognizing invading microorganisms by pathogen-associated molecular patterns (PAMPs). Binding of TLRs to PAMPs signals presence of microbial pathogens and triggers series of events that activate immune response. Thus, TLR ligands that mimic PAMPs and activate immune cells via TLRs are being developed for vaccine adjuvants to aid the generation of robust and long-lasting adaptive immune responses [52]. The principal ligands for TLRs 1, 2, 3, 5, 6, 7, and 9 were determined by gene targeting as early as between 1999 and 2003 [53]. A number of these ligands are now in clinical or late preclinical stages of development for multiple applications including vaccine adjuvants [54]. Among them, bacte- ria flagellin is very unique due to its protein property, which is recognized by cell surface TLR5, the only proteinbinding TLR that is conserved in vertebrates from fish to mammals. The flagellin molecule, consisting of conserved domains at the $\mathrm{N}$ terminus and $\mathrm{C}$ terminus and a middle hypervariable domain, can also be recognized by the cytosolic NLRC4 inflammasome receptor NAIP5 [55,56], though the contribution of this cytosolic pathway to the adjuvant activity of flagellin needs to be further defined [57]. In view of the substantial potency of flagellin as a potent activator of a broad range of cell types involved in innate and adaptive immunity, the potential of this fascinating protein seems to be almost unlimited for many applications [58].

\section{Enhancement of salivary SIgA antibodies and anti-caries protection by intranasal immuniza- tion with flagellin-PAc fusion protein}

The finding that flagellin from many bacteria species was a ligand for TLR5 further suggested its potential as an adjuvant [59]. Thereafter, an increasing number of studies have demonstrated the effectiveness and advantages of flagellin as a novel attractive adjuvant [58]. Furthermore, flagellin has also been proven to be an effective mucosal adjuvant in many vaccines against pathogens like Yersinia pestis, West Nile virus $[15,16]$. We have also demonstrated that recombinant FliC protein derived from Salmonella is an excellent mucosal adjuvant for enhancing local IgA responses [60].

Flagellin has a number of advantages for development of a promising adjuvant for use in human vaccines [58]. The most attractive advantage is the plasticity of flagellin for generation of fusion proteins of recombinant vaccine antigens and flagellin [61]. The fusion strategy can be achieved by inserting vaccine target antigen at the $\mathrm{N}$-terminus or C-terminus or within the hypervariable region of the flagellin. A number of vaccine candidates with this strategy have reached early stage clinical studies, and this represents one of the most promising new directions in vaccine development [13,14,17-19].

Based on the great capacity of flagellin to induce humoral and cellular immune responses, as well as mucosal immune response against foreign antigens, we explored the use of this molecule for the generation of fusion proteins containing caries vaccine antigens. We tried to insert the A-P fragment of PAc from $S$. mutans (rPAc) at the C-terminus of flagellin derived from $E$. coli (designated as $\mathrm{KF})$ to produce a single recombinant protein (KF-rPAc) in the $E$. coli expression system. It was demonstrated that KF-rPAc could promote significant higher rPAc-specific antibodies in serum as well as in saliva compared with the rPAc alone or mixture of rPAc and KF (KF+rPAc). Previous studies usually used at least $50 \mu \mathrm{g}$ dosage of PAc protein, chimeric protein SBR-GLU, or CTB-PAc coupled 
immunogen for inducing detectable mucosal immune responses or protective salivary IgA antibody against $S$. mutans infection in rats $[62,63]$. By flagellin-PAc fusion strategy, $4.2 \mu \mathrm{g}$ of KF-rPAc, which is equivalent to $2.5 \mu \mathrm{g} \mathrm{rPAc}$, could induce high levels of immune responses; and $8.5 \mu \mathrm{g}$ KF-rPAc could induce significantly elevated antibody responses and confer effective reduction of dental caries in rats. This fusion strategy of rPAc antigen with flagellin significantly reduced vaccine dosage. The most impressive result is that intranasal immunization of $8.5 \mu \mathrm{g}$ KF-rPAc achieved $64.2 \%$ reduction of dental caries in rats [21]. This demonstrated that flagellin and PAc fusion strategy is promising for anti-caries vaccine development, and KF-rPAc could be a good candidate of anti-caries mucosal vaccine.

However, the very potent antigenicity of flagellin itself led to a concern that immunity to flagellin might affect the potency of this molecule and induce possible side effects when used as a mucosal adjuvant [64], although there were reports that prior immunity to flagellin does not impair its adjuvant activity and does not lead to serious systemic effects [15]. We tried to replace the main antigenicity region domains D2 and D3 of flagellin with rPAc and successfully obtained a soluble KFD-rPAc recombinant protein. As we expected, KFD-rPAc reduced by more than $95 \%$ of the immunogenicity against full-length flagellin KF protein [65]. Surprisingly, this replacement of domains D2 and D3 in flagellin could induce high IgA-biased antibody responses at different mucosal sites and far fewer flagellin-induced systemic inflammatory responses [65]. The propensity to produce IgA-biased responses in mucosal sites especially in oral cavity is important and promising for caries mucosal vaccines.

\section{Development of a nasal-spray or nasal-drop anti-caries vaccine for human use}

Although the requirement for the physical linking of flagellin to the antigen is still a matter of debate, our observation showed that the replacement of the whole hypervariable region D2 and D3 of flagellin with vaccine antigen promotes mucosal $\operatorname{IgA}$ production and attenuates flagellin-induced inflammatory response after intranasal immunization [65]. The reason why the whole hypervariable region D2 and D3 of flagellin with vaccine antigen has propensity to produce IgA-biased responses in mucosal sites is intriguing and worth further exploration. The practical use of this strategy is essential for development of an effective and safe anticaries vaccines. To our knowledge, the KF-rPAc [21] and KFD-rPAc (unpublished data) are two good prototypes of anti-caries intranasal vaccines, which are easy for recombinant protein production in $E$. coli expression system at a large scale and low product cost for commercial use. The use of recombinant fusion flagellins containing PAc inserted into hypervariable region may be a simple and inexpen- sive way to enhance antigenicity of PAc for nasal immunization. The fact that recombinant flagellins have reached Phase I/II clinical trials should accelerate further studies in this direction $[19,66]$.

Considering the closer anatomical relationship to the oral cavity and the convenience for vaccine administration, intranasal immunization is likely to be the first choice for caries vaccine, which targets the nasal-associated lymphoid tissue (NALT). Caries vaccines can be administered in a nasal drop or nasal spray rather than in a hypodermic needle. Kids may choose nasal immunization more gladly over the formidable needles that deliver other vaccines. Intranasal caries vaccines, directed to key components of mutans streptococcal colonization and enhanced by safe and effective mucosal adjuvants and optimal delivery vehicles, are likely to be forthcoming [11]. However, some other elements should also be taken into account.

As dental caries usually develops slowly and occurs throughout life, immune protection would need to be long-lasting. Thus, the duration and anamnestic recall of salivary antibody responses are important factors. It is now clear that mucosal vaccination can induce both memory $\mathrm{IgA}^{+}$and memory $\operatorname{IgG}^{+} \mathrm{B}$ cells after oral, intranasal and intravaginal vaccinations [67]. Studies showed protection against infection induced by mucosal vaccines can last for several years, even though intestinal $\operatorname{IgA}$ responses vanish after 6-9 months [68-70]. For caries vaccines, the duration of salivary IgA responses induced by mucosal immunization need to be explored further. However, the longer duration of salivary IgA responses and related protection can be achieved by a renewed exposure to the mutans streptococcal or boost immunization to elicit a rapid recall response and thereby to sustain the capacity for preventing infection $[68,71]$.

Given that oral colonization with mutans streptococci mainly occurs during a 'window of infectivity' at around two years of age after primary teeth begin to erupt, it would be necessary to immunize infants or young children in order to provide immune protection prior to initial colonization with mutans streptococci [43]. For infants or young children, the safety of the immunization is the first priority. KF-rPAc and KFD-rPAc are good and safe choice for a preventive caries vaccine for infants and young children, in which the salivary $\operatorname{IgA}$ responses only inhibited oral colonization and biofilm formation of mutans streptococci but did not disturb growth of oral flora (unpublished data). Another alternative safe way for infants is to immunize young mothers intranasally for reducing their oral load of mutans streptococci and thereby diminishing the probability and extent of transmission to their infants. On the other hand, this way will induce the generation of antibodies to mutans streptococci in breast-milk and passively transfer IgA antibodies to protect infants at earlier stage through breast-feeding, though the protection effect needs to be demonstrated further [43].

Due to the current high caries prevalence rate all over the 
world, for juvenile and adults who have been already infected by mutans streptococci, a therapeutic anti-caries vaccine is an urgent need. To be able to clear micro-organisms from the oral cavity by antibody-mediated aggregation after colonization and accumulation of mutans streptococci, multiple specific antibodies to block the glucan-binding domains of GBPs and GTF, or inactivate GTF enzymes responsible for glucan formation might be also needed for enhancement of the antimicrobial activity by synergism. At present, there is no one-fit-all vaccine for any disease. Clearly, several possible dental caries vaccine approaches may have application in clinical trials. Finally, clinical trials of immunologically superior dental caries vaccine formulations will determine their usefulness for public health applications ultimately.

This work was supported by the National Key Research and Development Program, Ministry of Science and Technology of China (2007BAI28B04) and the National Science and Technology Major Project on Major Infectious Diseases (2012ZX10001-008, 2008ZX10001-010). I sincerely thank Dr. George Dacai Liu for critical reading and comments of the article. I am thankful to Drs. Dihan Zhou and Ying Sun for valuable assistance in the reference checking and editing. The author declares no potential conflict of interest with respect to the authorship and/or publication of this article.

1 Hajishengallis G, Russell M W, Michalek S M. Comparison of an adherence domain and a structural region of Streptococcus mutans antigen I/II in protective immunity against dental caries in rats after intranasal immunization. Infect Immun, 1998, 66: 1740-1743

2 Hamada S, Slade H D. Biology, immunology, and cariogenicity of Streptococcus mutans. Microbiol Rev, 1980, 44: 331-384

3 Loesche W J. Role of Streptococcus mutans in human dental decay. Microbiol Rev, 1986, 50: 353-380

4 Saekel R. Oral health data from China and their relevance for Germany. Prophylaxe Impuls, 2010, 14: 6

5 Shang X H, Li D L, Huang Y, et al. Prevalence of dental caries among preschool children in Shanghe County of Shandong Province and relevant prevention and treatment strategies. Chin Med J (Engl), 2008, 121: 2246-2249

6 Qi X Q. Report of the Third National Oral Health Survey. Beijing: People's Medical Publishing House, 2008

7 Fan $M$ W, Bian Z, Peng Z X, et al. A DNA vaccine encoding a cell-surface protein antigen of Streptococcus mutans protects gnotobiotic rats from caries. J Dent Res, 2002, 81: 784-787

8 Jia R, Guo J H, Fan M W, et al. Immunogenicity of CTLA4 fusion anti-caries DNA vaccine in rabbits and monkeys. Vaccine, 2006, 24: 5192-5200

9 Lewis D J, Huo Z, Barnett S, et al. Transient facial nerve paralysis (Bell's palsy) following intranasal delivery of a genetically detoxified mutant of Escherichia coli heat labile toxin. PLoS ONE, 2009, 4: e6999

10 Liang S, Hosur K B, Nawar $\mathrm{H} \mathrm{F}$, et al. In vivo and in vitro adjuvant activities of the B subunit of Type IIb heat-labile enterotoxin (LT-IIb-B5) from Escherichia coli. Vaccine, 2009, 27: 4302-4308

11 Smith D J. Prospects in caries vaccine development. J Dent Res, 2012 , 91: 225-226

12 Xu Q A, Yu F, Fan M W, et al. Protective efficacy of a targeted anti-caries DNA plasmid against cariogenic bacteria infections. Vaccine, 2007, 25: 1191-1195

13 Adar Y, Singer Y, Levi R, et al. A universal epitope-based influenza vaccine and its efficacy against H5N1. Vaccine, 2009, 27: 2099-2107

14 Bargieri D Y, Leite J A, Lopes S C, et al. Immunogenic properties of a recombinant fusion protein containing the C-terminal $19 \mathrm{kDa}$ of Plasmodium falciparum merozoite surface protein-1 and the innate immunity agonist FliC flagellin of Salmonella typhimurium. Vaccine, 2010, 28: 2818-2826

15 Honko A N, Sriranganathan N, Lees C J, et al. Flagellin is an effective adjuvant for immunization against lethal respiratory challenge with Yersinia pestis. Infect Immun, 2006, 74: 1113-1120

16 McDonald W F, Huleatt J W, Foellmer H G, et al. A West Nile virus recombinant protein vaccine that coactivates innate and adaptive immunity. J Infect Dis, 2007, 195: 1607-1617

17 Song L, Zhang Y, Yun N E, et al. Superior efficacy of a recombinant flagellin: H5N1 HA globular head vaccine is determined by the placement of the globular head within flagellin. Vaccine, 2009, 27: 5875-5884

18 Taylor D N, Treanor J J, Strout C, et al. Induction of a potent immune response in the elderly using the TLR-5 agonist, flagellin, with a recombinant hemagglutinin influenza-flagellin fusion vaccine (VAX125, STF2.HA1 SI). Vaccine, 2011, 29: 4897-4902

19 Treanor J J, Taylor D N, Tussey L, et al. Safety and immunogenicity of a recombinant hemagglutinin influenza-flagellin fusion vaccine (VAX125) in healthy young adults. Vaccine, 2010, 28: 8268-8274

20 Shi W, Li Y H, Liu F, et al. Flagellin enhances saliva IgA response and protection of anti-caries DNA vaccine. J Dent Res, 2012, 91: 249-254

21 Sun Y, Shi W, Yang J Y, et al. Flagellin-PAc fusion protein is a highefficacy anti-caries mucosal vaccine. J Dent Res, 2012, 91: 941-947

22 Fejerskov O. Changing paradigms in concepts on dental caries: consequences for oral health care. Caries Res, 2004, 38: 182-191

23 Scheie A A, Petersen F C. The biofilm concept: consequences for future prophylaxis of oral diseases? Crit Rev Oral Biol Med, 2004, 15: 4-12

24 Featherstone J D. The continuum of dental caries-evidence for a dynamic disease process. J Dent Res, 2004, 83: C39-42

25 Gambhir R S, Singh S, Singh G, et al. Vaccine against dental caries-an urgent need. J Vaccines Vaccin, 2012, 3: 136

26 McGhee, Michalek S M, Webb J, et al. Effective immunity to dental caries: protection of gnotobiotic rats by local immunization with Streptococcus mutans. J Immunol, 1975, 114: 300-305

27 Talbman M A, Smith D J. Effects of local immunization with Streptococcus mutans on induction of salivary immunoglobulin A antibody and experimental dental caries in rats. Infect Immun, 1974, 9: 1079-1091

28 Koga T, Yamashita Y, Nakano Y, et al. Surface proteins of Streptococcus mutans. Dev Biol Stand, 1995, 85: 363-369

29 Kuramitsu H K. Virulence factors of mutans streptococci: role of molecular genetics. Crit Rev Oral Biol Med, 1993, 4: 159-176

30 Guo J H, Jia R, Fan M W, et al. Construction and immunogenic characterization of a fusion anti-caries DNA vaccine against PAc and glucosyltransferase I of Streptococcus mutans. J Dent Res, 2004, 83: 266-270

31 Jenkinson H F, Lamont R J. Streptococcal adhesion and colonization. Crit Rev Oral Biol Med, 1997, 8: 175-200

32 Sun J, Yang X, Xu Q A, et al. Protective efficacy of two new anti-caries DNA vaccines. Vaccine, 2009, 27: 7459-7466

33 Smith D J. Dental caries vaccines: prospects and concerns. Crit Rev Oral Biol Med, 2002, 13: 335-349

34 Smith D J, King W F, Rivero J, et al. Immunological and protective effects of diepitopic subunit dental caries vaccines. Infect Immun, 2005, 73: 2797-2804

35 Wachsmann D, Klein J P, Scholler M, et al. Serum and salivary antibody responses in rats orally immunized with Streptococcus mutans carbohydrate protein conjugate associated with liposomes. Infect Immun, 1986, 52: 408-413

36 Smith D J, Taubman M A, Ebersole J L. Effects of local immunization with glucosyltransferase fractions from Streptococcus mutans on dental caries in hamsters caused by homologous and heterologous serotypes of Streptococcus mutans. Infect Immun, 1978, 21: $843-851$

37 Li F, Michalek S M, Dasanayake A P, et al. Intranasal immunization 
of humans with Streptococcus mutans antigens. Oral Microbiol Immunol, 2003, 18: 271-277

38 Smith D J, Taubman M A. Ontogeny of immunity to oral microbiota in humans. Crit Rev Oral Biol Med, 1992, 3: 109-133

39 Smith D J, Taubman M A. Emergence of immune competence in saliva. Crit Rev Oral Biol Med, 1993, 4: 335-341

40 Smith D J, Mattos-Graner R O. Secretory immunity following mutans streptococcal infection or immunization. Curr Top Microbiol Immunol, 2008, 319: 131-156

41 Lehner T. Immunology of Oral Diseases. Oxford: Blackwell Scientific Publications, 1992

42 Marcotte H, Lavoie M C. Oral microbial ecology and the role of salivary immunoglobulin A. Microbiol Mol Biol Rev, 1998, 62: 71-109

43 Russell M W, Childers N K, Michalek S M, et al. A caries vaccine? The state of the science of immunization against dental caries. Caries Res, 2004, 38: 230-235

44 Smith D J, Taubman M A. Oral immunization of humans with Streptococcus sobrinus glucosyltransferase. Infect Immun, 1987, 55: 2562-2569

45 Smith D J, Taubman M A. Effect of local deposition of antigen on salivary immune responses and reaccumulation of mutans streptococci. J Clin Immunol, 1990, 10: 273-281

46 Michalek S M, Childers N K. Development and outlook for a caries vaccine. Crit Rev Oral Biol Med, 1990, 1: 37-54

47 Ogra P L M J, Lamm M E, Strober W, et al. Mucosal Immunology. San Diego: Academic Press, 1999

48 Russell M W, Hajishengallis G, Childers N K, et al. Secretory immunity in defense against cariogenic mutans streptococci. Caries Res, 1999, 33: 4-15

49 Borges O, Lebre F, Bento D, et al. Mucosal vaccines: recent progress in understanding the natural barriers. Pharm Res, 2010, 27: 211-223

50 Kiyono H, Fukuyama S. NALT-versus Peyer's-patch-mediated mucosal immunity. Nat Rev Immunol, 2004, 4: 699-710

51 Fontana M, Dunipace A J, Stookey G K, et al. Intranasal immunization against dental caries with a Streptococcus mutans-enriched fimbrial preparation. Clin Diagn Lab Immunol, 1999, 6: 405-409

52 Beutler B A. TLRs and innate immunity. Blood, 2009, 113: 1399-1407

53 Akira S, Takeda K. Toll-like receptor signalling. Nat Rev Immunol, 2004, 4: 499-511

54 Coffman R L, Sher A, Seder R A. Vaccine adjuvants: putting innate immunity to work. Immunity, 2010, 33: 492-503

55 Lightfield K L, Persson J, Brubaker S W, et al. Critical function for Naip5 in inflammasome activation by a conserved carboxy-terminal domain of flagellin. Nat Immunol, 2008, 9: 1171-1178

56 Zhao Y, Yang J, Shi J, et al. The NLRC4 inflammasome receptors for bacterial flagellin and type III secretion apparatus. Nature, 2011, 477:
596-600

57 Vijay-Kumar M, Carvalho F A, Aitken J D, et al. TLR5 or NLRC4 is necessary and sufficient for promotion of humoral immunity by flagellin. Eur J Immunol, 2010, 40: 3528-3534

58 Mizel S B, Bates J T. Flagellin as an adjuvant: cellular mechanisms and potential. J Immunol, 2010, 185: 5677-5682

59 Hayashi F, Smith K D, Ozinsky A, et al. The innate immune response to bacterial flagellin is mediated by Toll-like receptor 5. Nature, 2001, 410: 1099-1103

60 Liu F, Yang J, Zhang Y, et al. Recombinant flagellins with partial deletions of the hypervariable domain lose antigenicity but not mucosal adjuvancy. Biochem Biophys Res Commun, 2010, 392: 582-587

61 Huleatt J W, Jacobs A R, Tang J, et al. Vaccination with recombinant fusion proteins incorporating Toll-like receptor ligands induces rapid cellular and humoral immunity. Vaccine, 2007, 25: 763-775

62 Katz J, Harmon C C, Buckner G P, et al. Protective salivary immunoglobulin A responses against Streptococcus mutans infection after intranasal immunization with $\mathrm{S}$. mutans antigen I/II coupled to the B subunit of cholera toxin. Infect Immun, 1993, 61: 1964-1971

63 Zhang P, Jespersgaard C, Lamberty-Mallory L, et al. Enhanced immunogenicity of a genetic chimeric protein consisting of two virulence antigens of Streptococcus mutans and protection against infection. Infect Immun, 2002, 70: 6779-6787

64 Nempont C, Cayet D, Rumbo M, et al. Deletion of flagellin's hypervariable region abrogates antibody-mediated neutralization and systemic activation of TLR5-dependent immunity. J Immunol, 2008, 181: 2036-2043

65 Yang J, Zhong M, Zhang Y, et al. Antigen replacement of domains D2 and D3 in flagellin promotes mucosal $\operatorname{IgA}$ production and attenuates flagellin-induced inflammatory response after intranasal immunization. Hum Vaccin Immunother, 2013, 9: 1-9

66 ClinicalTrials.gov. Comparative safety and immunogenicity of $1.0 \mu \mathrm{g}$ intramuscular (i.m.) and $2.0 \mu \mathrm{g}$ subcutaneous (s.c.) dosing with VAX102 (M2e-flagellin) universal influenza vaccine in healthy adults. 2009. http://www.clinicaltrials.gov/ct2/show/NCT00921947?term= influenza+and+universal\&rank=2

67 Lycke N. Recent progress in mucosal vaccine development: potential and limitations. Nat Rev Immunol, 2012, 12: 592-605

68 Brandtzaeg P. Induction of secretory immunity and memory at mucosal surfaces. Vaccine, 2007, 25: 5467-5484

69 Czerkinsky C, Holmgren J. Enteric vaccines for the developing world: a challenge for mucosal immunology. Mucosal Immunol, 2009, 2: 284-287

70 Holmgren J. Actions of cholera toxin and the prevention and treatment of cholera. Nature, 1981, 292: 413-417

71 Plotkin S A. Correlates of protection induced by vaccination. Clin Vaccine Immunol, 2010, 17: 1055-1065

Open Access This article is distributed under the terms of the Creative Commons Attribution License which permits any use, distribution, and reproduction in any medium, provided the original author(s) and source are credited. 